\title{
FRONTERAS Y FRONTEROLOGÍA: REFLEXIONES SOBRE LOS DESAFÍOS DE SU PROCESO DE ENSEÑANZA-APRENDIZAJE
}

\author{
TONY PAYAN \\ Professor da Universidade de Rice e da Universidade Autônoma de Ciudad Juárez \\ tony.payan@rice.edu \\ IRASEMA CORONADO \\ Professora da Universidade Estatal de Arizona (UEA) \\ irasema.coronado@asu.edu
}

\section{El nacimiento de una meta-disciplina}

Los estudios fronterizos son una meta-disciplina de reciente desarrollo ${ }^{1}$. La Asociación de Estudios Fronterizos, organización que agrupa a los estudiosos de las fronteras de muchas partes del mundo, por ejemplo, de suma importancia para la integración de la meta-disciplina, fue fundada apenas en $1976^{2}$. A casi cincuenta años de su conformación, la fronterología - un término también de reciente acuñación que aparece por primera vez, según una búsqueda del mismo, en el texto de Nweihed (1990) - es una metadisciplina todavía llena de meandros propios del desarrollo de cualquier campo académico novedoso. La novedad por supuesto impone una ruta accidentada en cualquier desarrollo disciplinar, pero también presenta oportunidades que permiten contemplar

1 Una meta-disciplina se caracteriza por la resistencia a limitarse a una sola disciplina-la práctica de la indisciplina, sin la cual raramente hay conocimientos nuevos; la premisa que la realidad avanza más rápidamente que el conocimiento de la realidad, lo cual requiere el uso constante de diferentes enfoques epistemológicos; los choques dialécticos entre lo de antes y lo de hoy y distintas cosmovisiones de la realidad; la fragmentación de la realidad, lo que requiere una construcción y una reconstrucción contante de la misma; el diálogo entre la experiencia personal y el método; el pluralismo como método; la superior de la complementariedad de unas disciplina con otras; y la observación constante de una realidad que se desmorona continuamente. Es en este sentido que este ensayo retoma el tema de los estudios fronterizos como una meta-disciplina (Torres Carral, 2006).

2 La Asociación de Estudios Fronterizos tiene una página de Internet, en la cual se pueden consultar sus actividades pasadas y recientes. Ver: https://absborderlands.org/.

Publicado em: Dorfman, A.; Filizola, R.; Félix, J. M. (Orgs.) Ensinando Fronteiras: projetos estatais, representações sociais e interculturalidade. Porto Alegre: Editora Letra1; Editora Diadorim, 2021, p. 106-134. doi: 10.21826/9786599023460-05 
fenómenos culturales, sociales, políticos, y económicos desde perspectivas con pocos engreimientos y presunciones. Además, el crecimiento disciplinar permite también contemplar fenómenos complejos desde el lente del objeto de estudio mismo - por ejemplo, la relación del fenómeno como tal con la historia, la geografía y el territorio, el imperialismo, la colonización, el nacionalismo, etc., así como también su relación con temas más acotados como el medio ambiente, la seguridad, los flujos migratorios, la cooperación en la gestión del territorio, la infraestructura binacional, etc.

Tomar un objeto de estudio, como las fronteras, y ponerlo al centro de la perspectiva, revela asimismo dimensiones nuevas de la organización social y humana. Permite una mirada crítica fresca de temas como el poder (Newman, 2003), la ciudadanía (Ambrosini et al., 2019), el privilegio (Park; Pellow, 2019), los mercados negros (Díaz, 2015), etc. Esto es en parte posible porque lo que une a los fronterólogos es no sólo un complejo objeto de estudio que exige una constante redefinición - las fronteras y todos sus conceptos auxiliares y sus problemáticas - sino porque los estudiosos vienen de todas las disciplinas y se convierten, por necesidad e interacción, en investigadores multidisciplinarios, cuyo conocimiento individual y disciplinar complementa al de los demás miembros de la comunidad epistémica. El camino de la fronterología es uno en parte en desarrollo y en parte consolidado, pero también en parte improvisado, aunque siempre enormemente rico porque se alimenta de muchos elementos teóricos y conceptuales, de muchas aproximaciones metodológicas, y de muchas áreas del conocimiento humano.

El carácter relativamente nuevo y multifacético de la meta-disciplina de los estudios fronterizos significa sin embargo que existen retos y problemas importantes en el estudio del objeto propiamente - las fronteras y los espacios fronterizos - pero también existen retos y problemas que afectan sin duda el proceso de enseñanza-aprendizaje de quienes imparten clases y de quienes aprenden sobre las fronteras y sus fenómenos circundantes. Un examen de múltiples programas de estudio sobre fronteras en Europa, Norteamérica, y Latinoamérica revela, por ejemplo, que quienes estudian y enseñan sobre las fronteras enfrentan todavía numerosos retos teóricos y conceptuales y metodológicos, al mismo tiempo que enfrentan una serie de desafíos éticos, materiales, 
y, por supuesto, didácticos. Este inventario de programas de estudio incluyó universidades tan diversas como la Universidad de Victoria y la Universidad de Montréal en Québec en Canadá; Surco Oaxaca, la Universidad Autónoma de Ciudad Juárez, El Colegio de la Frontera, FLACSO, y la Universidad Autónoma de Baja California, en México; Earlham College, Arizona State University, Berkeley University, Columbia University, Stanford University, San Diego State University, Rutgers University, y la Universidad de Texas en El Paso, en los Estados Unidos; The University of Eastern Finland, Central European University, The University of Birmingham, York University and Trinity College, en Europa. El número de cursos y programas en estudios fronterizos continúa creciendo, sin embargo, y queda pendiente en el futuro estudiar qué se enseña y cómo.

Este ensayo aborda algunos de estos retos y desafíos, fundamentalmente en su relación con el proceso de enseñanza-aprendizaje en la fronterología. Se ubica en la intersección entre la investigación fronterológica y la incidencia de sus retos y desafíos sobre los procesos de enseñanza-aprendizaje. Las preguntas centrales que nos mueven a esta reflexión son pues, por un lado: ¿Cuáles son los problemas más sustantivos que enfrentan quienes dedican gran parte de su tiempo, recursos, y energía a producir e impartir conocimientos relacionados con las fronteras? y, por otro lado, ¿cómo inciden estos en la inducción de estudiantes de pregrado y postgrado a los estudios fronterizos?

Para contestar estas interrogantes, este ensayo toma, en principio, los retos internos de la meta-disciplina, tales como los procesos de conceptualización del objeto de estudio, los desafíos metodológicos, y los problemas teóricos, desglosando los que éstos representan para la investigación y docencia de la fronterología, y luego los retos externos que presenta el trabajo de campo en los espacios fronterizos en el mundo de hoy, tales como la accesibilidad al objeto de estudio, los costos del trabajo de campo, la dificultad de desplazamientos hacia el objeto de estudio, etc. El argumento central es que quienes se dedican a la investigación y docencia en los estudios fronterizos enfrentan retor significativos que emanan no sólo de la práctica de la enseñanza-aprendizaje propiamente sino también de su relación con el objeto de estudio y los acerca- 
mientos teóricos y metodológicos, muchos de los cuales todavía están por resolverse dentro de la propia meta-disciplina. Conocer cuáles son estos desafíos, sin embargo, es esencial para resolver el problema de la fronterología como materia de estudio científico tanto en el campo como en el aula. En este sentido, poseer una conciencia integral de estos retos y desafíos sólo puede ayudar a lograr una mayor efectividad en la investigación y docencia enfocadas en la fronterología.

\section{Estudiando el estudio de las fronteras}

Llevamos a cabo una búsqueda por Google usando las palabras "syllabus" y "border" y "programas de estudio" y "frontera". Encontramos numerosos programas de estudios y aunque este es un número pequeño, nos dio una idea de la amplio y diverso que es el estudio de la frontera. Unos programas de estudio se enfocan en temas como migración, salud pública en la frontera, energía, gobernanza. Otros programas de estudio incorporan temas más interdisciplinarios y sólo dos usaron una perspectiva comparativa. La mayoría de los programas de estudio que consultamos tratan con la frontera México - Estados Unidos. Encontramos que unos profesores piden que los estudiantes lleven a cabo trabajos más aplicados. Por ejemplo, un programa de estudio pedía que el estudiante identificara un problema en la frontera y desarrollara un resumen de políticas públicas para presentarle a personas con cargo político y en posición de tomar decisiones políticas. Otro programa de estudio pedía también un resumen de políticas públicas, pero en un contexto binacional. Las lecturas asignadas varían también, $\mathrm{y}$, aunque buscamos lecturas comunes, en realidad había una gran diversidad de autores y artículos, unos muy especializados y otros más interdisciplinarios.

\section{Retos conceptuales de la fronterología}

\section{y el proceso de enseñanza-aprendizaje de las fronteras}

Muchos estudiosos de las fronteras se hacen ab initio una pregunta aparentemente sencilla, pero que, sin duda, es bastante aguda: ¿Cuál es el objeto de los estudios fronterizos? Esta pregunta, a pesar de su aparente sencillez, es sumamente compleja. Para responderla, 
desde una perspectiva pedagógica, nos remitimos a revisar documentos utilizados en los procesos de enseñanza-aprendizaje (programas de estudios) y de contenido de las reuniones y conferencias de fronterólogos. Esta revisión incluyó el análisis de varios programas de estudios sobre fronteras, tanto de pregrado como de posgrado, disponibles en la internet, así como las memorias de congresos sobre fronteras de los últimos quince años (especialmente las de la Asociación de Estudios Fronterizos o ABS, de la Red Border Regions in Transition o BRIT y algunas conferencias ocasionales). Este ejercicio del contenido y acercamiento a tema de las fronteras evidencia no sólo que la mayor parte de ellos bregan con esta compleja interrogante de muy diversas maneras, y también que no hay consenso sobre la manera de abordar el objeto de estudio de manera uniforme.

Muchos programas de estudio, por ejemplo, comienzan con una discusión básica sobre la definición de fronteras, mientras otros comienzan con una perspectiva crítica del tema de las fronteras. Todos, sin embargo, revelan que la respuesta a la pregunta central - ¿qué son las fronteras? - es sumamente compleja y varía dependiendo de quién la responda. Y si bien la respuesta más llana consiste en nombrar los límites físicos entre países, las zonas próximas a los puntos limítrofes, y los sistemas sociales, políticos y económicos que dividen y simultáneamente unen a dos naciones, dos territorios, dos demarcaciones jurisdiccionales, etc., los problemas definicionales del tema se abordan diferentemente en Europa-con un acercamiento más teórico; en Norteamérica, en donde el tema se problematiza en gran parte basado en la asimetría entre Estados Unidos y sus vecinos; en África, en donde la experiencia colonial es central; en el Medio Oriente, donde el radicalismo musulmán trasciende los esquemas territoriales o el conflicto con Israel domina la visión del problema; o Latinoamérica, en donde los problemas de identidad siguen presentes a la par de fronteras abiertas y a menudo en disputa.

En otras palabras, la manera en que los programas de estudio abordan las fronteras y la manera en que estos temas se discuten en los congresos sobre las fronteras y zonas fronterizas apunta a la diversidad con la que los fronterólogos abordan el tema y, legítimamente, que lo hacen a partir de sus experiencias históricas, cultu- 
rales, y geográficas. Es decir, lo que este análisis de los documentos que se van produciendo en la meta-disciplina demuestra es que el problema de las fronteras es fundamentalmente contextual, y esto complica la labor docente porque impartir cursos sobre fronteras implica tender puentes entre la universalidad del objeto de estudio (por ejemplo, la demarcación limítrofe) y su contexto (la experiencia del imperialismo o la colonización o la historia de conflicto identitario, etc.). Erigir puentes entre la construcción de la universalidad conceptual de las fronteras y sus particularidades se convierte así en un reto fundamental del proceso de enseñanza-aprendizaje.

Evidentemente, la historia, la cultura y la geografía importan mucho e inciden en las condiciones del objeto de manera individual-procesos que nos llevan a preguntarnos quiénes habitaban ahí antes, qué sucedió con estos aborígenes cuando las demarcaciones se impusieron; cuáles son las asimetrías que se presentan de un lado y otro; qué problemas identitarios se hacen visibles; etc., y la manera en que impartimos clases y llevamos a cabo las conversaciones académicas del objeto de estudio. En otras palabras, cada programa de estudio aborda el tema fusionando la universalidad del objeto y dando contexto propio al mismo. En el estudio y aprendizaje sobre fronteras, esta encrucijada es inevitable-lo cual queda demostrado tanto en los programas de estudio de numerosas universidades del mundo como en las mesas de trabajo de las conferencias y congresos de fronterólogos.

Ahora bien, por algún lado se ha de comenzar y, por tanto, una demostración de mapas a veces acompaña este tipo de respuestas-una perspectiva cartográfica, por así decirlo, la cual, sin duda, tiene un valor importante y muestra la íntima y complicada relación entre los mapas y las fronteras (Nikolic, 2019; Diener; Hagen, 2010). La relación que guarda el concepto de frontera desde la cartografía ha adquirido enorme importancia (Melo Ribeiro, 2017), con amplia razón. Una definición territorial, o cartográfica, de las fronteras, sin embargo, es cada vez más improcedente, porque circunscribe los estudios fronterizos a algo material, precisamente cuando las fronteras adquieren significaciones subjetivas y se internalizan en los Estados-nación-como es el caso de las fronteras identitarias (i.e., lo que significa ser catalán, español y eu- 
ropeo al mismo tiempo). Muy rápidamente, la mayor parte de los programas de estudios abordan el objeto desde perspectivas tanto contextuales como críticas. Lo mismo sucede en las reuniones de la disciplina. Las mesas de trabajos sobre fronteras en múltiples programas analizados revelan que los problemas conceptuales de la fronterología son engañosamente simples.

Esta observación sobre la relación entre la fronterología y la cartografía o entre la fronterología y otras disciplinas que abastecen sus discusiones, tales como la economía, la ciencia política, o la sociología, no pretende restarle importancia a la relación del concepto de frontera con las manifestaciones físicas del mismosu contexto geográfico, histórico, étnico, etc. Evidentemente, y raramente, se puede pensar en una frontera sin un punto geográfico de referencia o sin los sistemas y subsistemas históricos, étnicos, geográficos, económicos, políticos o sociales empotrados en esos espacios. A pesar del enorme valor de la manifestación visual de las fronteras en la meta-disciplina de los estudios fronterizos, afortunadamente, el concepto de frontera se ha ido ampliando significativamente. Es decir, aunque en cualquier introducción a los estudios fronterizos en la pedagogía moderna, el concepto de frontera comienza por la cartografía y la geopolítica (Sevastianov et al., 2015, p. 15-33), pasa por la economía, la ciencia política y sociología, los estudios de la frontera, como tal, y termina con el estudio crítico de los espacios fronterizos, incluyendo el colonialismo, el imperialismo, el poder, la identidad, etc., la diversidad de acercamientos al tema han ido adquiriendo mayor extensión y profundidad y una dimensionalidad mucho más rica. Así pues, debido al carácter caleidoscópico de las fronteras, desde finales del siglo XX, en el campo de los estudios fronterizos han surgido visiones críticas del estudio de las fronteras como objeto científico (Parker; Vaughan-Williams, 2009, 2012). Parker y Vaughan-Williams argumentan que la frontera no es algo que se manifiesta de manera simple y sencilla, de forma no mediada por otros fenómenos. No es algo simplemente "presente," ni tampoco como algo establecido de manera permanente, ni accesible de manera obvia. Al contrario, es un fenómeno multifacético y en un estado de evolución constante. Por esta razón, dicen, es algo que invita a ser explorado, investigado, con sus 
imaginarios fronterizos alternativos. De igual manera, Magyar-Haas (2012) argumenta que el concepto de frontera requiere considerar al cuerpo mismo de las personas que habitan en contextos fronterizos como una especie de frontera itinerante. Las personas llevan consigo, marcado en sus huellas, sus iris, sus rasgos faciales, el concepto de frontera (Zureik y Salter, 2013). De igual manera, Newman (2003) explora otros conceptos, como el ejercicio del poder, por ejemplo, que irradian del propio concepto y que fuerzan a entender la frontera como un concepto que carga con dimensiones múltiples (Rumford, 2012). Hay también quienes abordan las fronteras desde campos tan vastos como el de la lingüística y el arte (Melo Ribeiro, 2017). Así pues, las fronteras poseen un referente material-una línea, un territorio, una valla, pero también tienen contenido subjetivo que va más allá de los constructos que en las ciencias sociales son al mismo tiempo variables independientes y variables dependientes. La fronterología requiere también de acercamientos epistemológicos, estéticos, y hasta ontológicos. Y, por supuesto, la fronterología no está vacía de una interacción muy compleja con la cultura y su producción. El reto para el fronterólogo es pues abordar la enseñanza de las fronteras como un proceso sumamente complejo y muy propio de la vida humana.

Es decir, para prosperar, el proceso de enseñanza-aprendizaje enfocado en las fronteras debe considerar casi simultáneamente los procesos de construcción de la frontera y sus manifestaciones físicas, así como la territorialización de los espacios, los flujos y movimientos transfronterizos, los cambios del paisaje, la distribución de los recursos naturales, la habitabilidad de las zonas fronterizas y sus afectaciones, y los procesos de institucionalización y gobernanza propios de las fronteras-con todos sus esquemas de permisividad y opresión (Lamont y Molnár 2002), pero también el porqué ontológico y epistemológico de las fronteras, ya sean estas físicas o completamente subjetivas y móviles. Y esta multifacética visión de las fronteras complica su presentación en el salón de clases. Es difícil para un docente encapsular toda esta riqueza conceptual, todavía en plena discusión entre los fronterólogos, confinado en las cuatro paredes de un salón, con apenas el auxilio de imágenes y videos tomados de la Internet, pero también es prácticamente imposible andar por todo el mundo observando los 
matices más sutiles del concepto de frontera. El concepto no se presta para capturarlo de manera sencilla en un salón de clases, pero tampoco se presta para estudiarlo con un cierto nivel de inmediatez. La frontera fuerza al docente a representarla en todas sus dimensiones, pero siempre de una manera un tanto superficial, sobre todo si no es posible acceder fácilmente al campo. Al conformar un programa de estudios, es esencial pues que el instructor considere todas estas complejas dimensiones y encuentre los mejores y más efectivos métodos para transmitir la riqueza del concepto mismo en la realidad humana.

Así pues, el problema central de la enseñanza y el aprendizaje en materia de fronteras es cómo considerar el gran problema de la necesidad de impartir conocimientos universales al mismo tiempo que dar a conocer contextos muy particulares y conducir a las mentes de los estudiantes por conocimientos básicos al mismo tiempo que inculcar una cultural crítica de la fronterología.

\section{El reto de la multidisciplinariedad de los estudios fronterizos}

El apartado anterior deja además en claro que los estudios fronterizos son, inherentemente, multidisciplinarios. Es decir, los estudios fronterizos son una meta-disciplina, es decir un campo que, como se dijo, toma de muchas otras disciplinas - de la cartografía, de la geopolítica, de la administración pública, de la sociología, de la antropología, de las relaciones internacionales, de la economía, de la ingeniería, y hasta de la filosofía, etc. Y esto nos lleva a pensar que un fronterólogo debe, casi por necesidad, conocer de muchos campos para poder transmitir en el proceso de enseñanza-aprendizaje la complejidad y pluridisciplinariedad de los estudios de las fronteras. Pero ¿quién puede saber de todo esto de tal manera que lo pueda transmitir en un curso sobre fronteras? La exigencia sobre el conocimiento es muy vasta.

Dar cursos de fronterología es transmitir, aparte de la complejidad del concepto de frontera como tal, la perspectiva multidisciplinaria del mismo y esto presenta un enorme reto porque la respuesta a la pregunta ¿Cuál es el objeto de los estudios fronterizos? depende también de la disciplina desde la que se aborda el objeto de estudio - como en un prisma, cada ángulo revela algo diferente. En cierta 
manera, desde nuestras disciplinas todos podemos ser fronterólogos, pero un docente fronterólogo debe entender por lo menos un poco de todo - una tarea difícil si no imposible. Desde la geopolítica, la frontera se ve como territorios y conflicto (Van Houtum, 2005); desde la sociología como una serie de sistemas humanos, culturales e identitarios (Bhabha, 2013); desde la lingüística como una hibridación del lenguaje (Martínez, 2003); desde la economía como la estructura de incentivos de conducta que se manifiestan en los flujos de bienes, servicios, capital, y personas (Manyika et al., 2014; Van Schendel, 2005); desde la antropología en formas de vida ocasionadas por el fenómeno físico de la frontera (Han y Antrosio, 2019); y así sucesivamente. Claro que existen también otras formas de organizar el estudio de las fronteras: La línea fronteriza como una variable dependiente, como algo producido por otros fenómenos, i.e., colonización, capitalismo, y la expansión territorial (Herbst, 1989) o el desarrollo y la proximidad urbana (Boehmer y Peña, 2012), o como una variable independiente, productora de otros fenómenos. Todos estos elementos deben considerarse en la conducción del proceso de enseñanza-aprendizaje, el cual debe dejar en claro la direccionalidad de la técnica metodológica, es decir, si el estudio contempla a la frontera como un productor de fenómenos o la producción de otros fenómenos, so pena de confundir al estudiante y perder su atención. Otros estudiosos de la frontera encuentran la solución a este reto en las transversalidades interdisciplinarias del objeto, contemplándolo simultáneamente como variables dependiente e independiente, como esquemas complejos de los espacios fronterizos en los cuales no es posible discernir qué incluye sobre qué sino que todos los elementos del terreno interactúan de manera compleja; o como problemas de habitabilidad que surgen a partir de espacios divididos; o como una cuestión de flujos legales e ilegales que originan esquemas de control y vigilancia, etc. Al final, las fronteras son totalizantes en su influencia en todas las direcciones, y el trabajo de toda clase sobre ellas debe al mismo tiempo dejar clara su complejidad y hacer el objeto simple de entender.

En este sentido, muchos cursos de estudios fronterizos, según se refleja en los programas de estudios analizados para este ensayo, comienzan primero con una discusión profunda del objeto 
de los estudios fronterizos y su contenido se va dirigiendo al área de especialidad del docente, sin abordar muchos de los otros temas, en gran parte porque no son parte del acervo intelectual del enseñante. Dar clases de estudios fronterizos es pues, sin duda, un enorme reto para los docentes puesto que requiere de un conocimiento amplio en temas altamente diversos, aunque mayormente en las ciencias sociales. En los estudios fronterizos se conjuntan muchos temas, pero también muchas disciplinas y campos de estudio que requieren de una capacidad sintética vasta para poder involucrar al estudiante de manera exitosa en el proceso enseñanza-aprendizaje. Un reto auxiliar es también que, al estudiante de fronteras, incluyendo a los estudiantes de pregrado como de postgrado, se le debe exigir una preparación anterior profunda en muchos campos para poder examinar un objeto tan completo como las fronteras y participar activamente de las diversas discusiones sobre el tema en seminarios, talleres, simposios, y otras reuniones propias de la meta-disciplina. Una ventaja que ofrece el estudio de las fronteras, sin embargo, es que el objeto se presta también para socializar al estudiante en varias otras disciplinas. Es decir, al enseñar sobre el tema de las fronteras, se pueden también transmitir conocimientos en otras áreas, tales como la historia, la geografía, los estudios culturales, la economía, la política, la sociología, la antropología, etc.

\section{Retos metodológicos y su incidencia en la docencia sobre fronteras}

Como se mencionó anteriormente, los estudios fronterizos presentan una serie de retos al proceso de enseñanza-aprendizaje a partir de las discusiones y los debates internos de la meta-disciplina. Pero, además de estos, existen otros retos que tienen que ver con los instrumentos metodológicos propios de un campo tan abierto y multifacético que no sólo aún no resuelve la mayor parte de sus temas conceptuales y teóricos, sino que tampoco ha resuelto gran parte del problema de sus acercamientos metodológicos. Así pues, entre otros retos substantivos (o internos) de los estudios fronterizos se encuentran también los predicamentos metodológicos que afligen a la meta-disciplina. El Estado-nación, por ejemplo, sigue siendo un referente central en las metodologías utilizadas en la fronterología (Amelina et al., 2012). Además, los estudios fron- 
terizos se caracterizan fundamentalmente por ser de corte cualitativo - especialmente estudios de caso, con una riqueza descriptiva amplia, pero que limitan la capacidad de encontrar patrones universales dentro de la disciplina (Wilson y Donnan, 2012). Es decir, los estudios cuantitativos están prácticamente ausentes de los estudios fronterizos. Una revisión de la bibliografía, y también de los programas de estudio sobre temas fronterizos y de las discusiones en congresos y conferencias, muestra que efectivamente el sesgo ha sido fundamentalmente hacia los estudios de caso. Ahora bien, los estudios fronterizos se aventuran de vez en cuando a los estudios comparados, pero muchos de ellos carecen del rigor de los estudios comparados sistemáticos (Paasi, 2012; Correa-Cabrera y Konrad, 2020). En muchos programas de estudio, por ejemplo, se asignan lecturas sobre casos a profundidad, con pocos hilos comparativos sobre distintas fronteras entre sí. Los estudios cuantitativos están prácticamente ausentes. Hay incluso quienes han argumentado que uno de los impasses más importantes del desarrollo teórico de la meta-disciplina de los estudios fronterizos pasa precisamente por un debate sobre los métodos utilizados en la misma (Payan, 2014). Se hace urgente, sin embargo, que tanto en la producción substantiva como en el ejercicio de los procesos de enseñanza-aprendizaje, busquemos utilizar herramientas metodológicas que permitan comparar entre diversas interacciones transfronterizas, diversos sistemas económicos y los flujos que estos representan, los esquemas de cooperación binacional en diversos contextos, la hibridación cultural e identitaria que ocurre en las fronteras de muchos países, etc. Se requiere, además, que se busque activamente utilizar bases de datos para llevar a cabo estudios cuantitativos que permitan teorizar desde otras perspectivas metodológicas. Sin una diversidad de acercamientos metodológicos, la meta-disciplina no podrá avanzar en su autonomía como un campo independiente de otras disciplinas, aun cuando se encuentre en interacción con estas. Tampoco podremos construir la disciplina en el proceso de enseñanza-aprendizaje a partir de la instrucción en diversas metodologías que avancen las habilidades de los estudiantes e incluso de los mismos miembros de la comunidad epistémica. 
Independientemente de los temas alrededor de los instrumentos metodológicos más adecuados para la fronterología, el impacto de este debate es profundo en el proceso de enseñanza-aprendizaje porque la mayor parte de los estudios presentados en las conferencias $-\mathrm{y}$ por tanto en los salones de clase - son de corte cualitativo, altamente descriptivo, y muy tímidos en su manejo de bases de datos en general. Efectivamente, un vistazo por los programas de estudio sobre fronteras deja entrever el sesgo cualitativo de la mayor parte de los estudios sobre fronteras y la enorme necesidad no sólo de romper con los impasses teóricos sino también con el problema del método. Peor aún, los componentes metodológicos de los programas de estudio que revisamos raramente contienen un apartado dedicado a discutir cuáles son los mejores acercamientos metodológicos a la meta-disciplina.

Ahora bien, existe un enorme debate en los estudios fronterizos con respecto a la posibilidad de transcender los estudios de caso. Las fronteras son fenómenos únicos y es cierto que buscar acercamientos teóricos requiere a veces de una cantidad de datos no fácilmente disponible a los estudiantes de las fronteras. Es cierto también que encontrar bases de datos compatibles mundialmente es difícil, y que a veces hay que dedicar tiempo a generarlas - lo cual es también muy costoso. En efecto, los estudios estadísticos están ausentes en parte porque estos problemas básicos de medición no han sido resueltos (Gerber y Mendoza Cota, 2020). Pero esto no debe implicar que los estudiantes de fronteras deban ser confinados por los docentes a estudios cualitativos. $\mathrm{Al}$ contrario, los cursos de estudios fronterizos deben representar oportunidades importantes para inducir a los estudiantes a pensar en una diversidad de métodos o en acercamientos mixtos para lograr encontrar patrones alrededor del fenómeno de las fronteras y apresurar el paso de la meta-disciplina. Es claro que muchas otras disciplinas, por ejemplo, la ciencia política, pasaron por este proceso y su sesgo se convirtió en lo contrario - un sesgo cuantitativo en la mayor parte de los estudios de hoy. Por supuesto que no se trata de dar un giro hacia lo cuantitativo, pero se pierde un elemento importante si no se busca la utilización de métodos cuantitativos o mixtos en los estudios fronterizos. Ese sigue siendo el reto. 


\section{Investigación de campo en los espacios fronterizos}

Además de los importantes debates conceptuales, teóricos y metodológicos internos a la meta-disciplina de los estudios fronterizos, los cuales presentan enormes retos y problemas al proceso de enseñanza-aprendizaje, el análisis de las fronteras enfrenta también retos prácticos, propios de la importancia que han cobrado las fronteras ante la creciente ingobernabilidad mundial y el retroceso hacia modelos nacionalistas de gobernanza. En efecto, muchos de los problemas de gobernanza global se han agolpado en las franjas fronterizas, en donde concurren los grandes flujos asociados con los procesos de globalización, desde los migrantes expulsados por las condiciones económicas y de seguridad en sus países (Mavroudi y Nagel, 2016) y los deportados de los países desarrollados hacia los países en vías de desarrollo, hasta los flujos ilegales de las múltiples industrias ilegalizadas que van desde los estupefacientes y otros contrabandos (Roth, 2017) hasta las especies en peligro de extinción (Schneider, 2012) hasta los crecientes flujos comerciales y los millones de turistas. Las respuestas de los Estados-nación ha sido clara: Refronterizar dramáticamente sus límites, aumentando de manera considerable el andamiaje de seguridad, vigilancia y control en los mismos - con enormes costos a los erarios, a los usuarios de las fronteras, y a la libertad de circulación mediante la creación de espacios bajo estado de excepción. En los espacios fronterizos se manifiestan además las estructuras de poder - medidas en las prioridades de los estados adyacentes, en donde es evidente que mientras que las personas son indeseadas o no bienvenidas, el comercio de bienes y servicios cobra una prioridad sorprendente. Aun cuando los flujos de personas disminuyan, por ejemplo, los flujos impulsados por el privilegio del capital no lo hacen - tal y como lo hemos visto en la frontera México-Estados Unidos durante la pandemia de la COVID-19 en el año 2020. Así pues, durante el siglo XXI, los espacios fronterizos han experimentado un alto nivel de securitización (Popescu, 2011). Las fronteras se han convertido en espacios de amplias disputas en materia de migración, crimen organizado, y otros fenómenos indeseables desde el punto de vista de los gobiernos centrales, quienes han desplegado enormes presupuestos y cuerpos policíacos militarizados con el afán de controlar sus peri- 
ferias territoriales. El número de barreras físicas - en muchos casos muros fronterizos - se ha multiplicado también (Vallet, 2014). Y se han creado espacios de excepción, en los cuales el usuario del cruce fronterizo es obligado de jure y de facto a renunciar a cualquier derecho ciudadano e incluso humano por el simple hecho de cruzar la línea fronteriza (Salter, 2008; Davitti, 2019). En general, cualquier persona que se acerca a una línea fronteriza hoy arriesga ser expulsada de la zona, cuestionada sin apelación a ningún derecho, o incluso arrestada o detenida-y en algunos lugares se arriesga hasta la muerte como es el caso de la zona limítrofe entre Israel y Palestina, por ejemplo (Homes y Holder, 2019).

Ahora bien, ¿qué significa esto para los estudiosos de las fronteras? Los autores de este ensayo, estudiosos de la frontera México-Estados Unidos han sido en varias ocasiones ahuyentados de la franja fronteriza al investigar la infraestructura física y las actividades de la Patrulla Fronteriza de Estados Unidos. La franja adyacente se considera territorio fuera del alcance de los estudiosos-los activistas son todavía menos bienvenidos. El gobierno de Estados Unidos ha clasificado gran parte de las estadísticas y las actividades de las agencias gubernamentales que se hacen cargo de la frontera, so pretexto de arriesgar la seguridad de tales actividades. Los agentes de la Patrulla Fronteriza (BP), los cuales forman parte de la Agencia de Aduana y Protección Fronteriza (CBP), y la propia Agencia de Migración y Aduanas (ICE), entre otras, tienen acceso a información de los cuerpos de inteligencia e información sobre periodistas y activistas con el propósito de controlar el acceso a sus actividades (Del Bosque, 2019). La inmunidad de la que gozan por sus propios abusos de migrantes e incluso de investigadores y estudiantes legítimos y de activistas de las fronteras también ha crecido. En cierta manera, estudiar las fronteras es arriesgarse a caer en manos de estos agentes, cuya discrecionalidad es casi total, puesto que los espacios fronterizos son prácticamente zonas de excepción.

Todo esto ha significado una resistencia cada vez mayor sobre la capacidad de los académicos de obtener información, de supervisar las actividades de los agentes fronterizos, y de acceder a los espacios de la franja. Las burocracias son cada vez más resis- 
tentes a la capacidad de los estudiosos de las fronteras - y de los activistas - de revisar y observar sus acciones y movimientos. De manera más incisiva, las agencias gubernamentales comienzan a negar acceso a datos sobre los flujos y sobre sus propias acciones, lo cual dificulta estudiar los esquemas que toma la gobernanza de los espacios fronterizos y los flujos transfronterizos. Un número creciente de fronteras en el mundo han seguido esta trayectoria, dificultando el trabajo académico de la mayor parte de los estudiosos. Así pues, exigir trabajo de campo a nuestros propios estudiantes de fronteras implica exponerlos a ciertos peligros que si bien no son letales sí pueden involucrar acusaciones tales como la "interferencia" con actividades oficiales o el traspasar espacios que las propias agencias gubernamentales consideran exclusivas.

Es decir, el estado de excepción que se ha asentado en los espacios inmediatos a los límites fronterizos, la secrecía con que las burocracias ejercen el poder, el intimidante uso de la tecnología y la ley en contra de estudiosos y activistas (Jordan, 2019), el uso retórico y político de lo fronterizo, y la resistencia a rendir cuentas por parte quienes controlan esos espacios han hecho impráctico e incluso peligroso el trabajo de campo. Las visitas a la zona fronteriza son interrumpidas por agentes de la Patrulla Fronteriza averiguando porqué académicos y estudiantes están visitando el lugar en donde está el muro. Es común que los agentes pidan que la gente se retire del lugar "por su bien" porque dicen que es una zona de conflicto. Entrevistar a deportados, o a refugiados (en ambos lados de la frontera) también es más difícil porque los albergues limitan el acceso a visitantes para poder proteger a las personas bajo su cargo. Para los académicos que investigan asuntos binacionales es más difícil cruzar la frontera de regreso a los Estados Unidos y pueden perder 3-4 horas en las filas para regresar. Todo esto hace que el trabajo de campo sea más difícil, si no imposible. Para los académicos, como nosotros, que sabemos que los problemas binacionales requieren de soluciones binacionales, el no poder trabajar e investigar en ambos lados de la frontera es una gran limitante para poder documentar el fenómeno social. También, la falta de interacción binacional entre académicos, ciudadanos, 
Organizaciones no Gubernamentales, representantes de gobierno, impide la cooperación y el entendimiento transfronterizo que es fundamental en poder solucionar problemas fronterizos.

Ahora bien, las amenazas al trabajo de investigación de campo y la capacidad de hacerlo de manera segura no sólo vienen del Estado y del aparado de seguridad desplegado por el mismo Estado, sino que vienen también de los mismos grupos delincuenciales que también utilizan la frontera para sus actividades. Por las fronteras fluyen no sólo capitales, bienes y servicios e individuos debidamente sancionados por los gobiernos sino también capitales, bienes, servicios e individuos ilegalizados o no autorizados por los gobiernos. Como tal, estos flujos constituyen poderosos mercados negros que se nutren de los réditos ocasionados por el mismo proceso de ilegalización frente a una demanda que no se extingue simplemente porque el Estado lo ordena. Así pues, individuos que se dedican a facilitar y gobernar estos flujos subrepticios también tienen un interés en no ser detectados, descubiertos y mucho menos estudiados en sus actividades. Cualquier académico o estudiante de las fronteras que dedique parte de su trabajo de campo a entender estos flujos se expone también al peligro que representan los actores de estos mercados negros. Ahora bien, cada vez se ha hecho más evidente que en las fronteras operan actores criminales que en sí representan un peligro para los investigadores de campo, pero también agentes de BP, CBP o ICE o cuerpos policiales - dependiendo de la frontera - que colaboran con grupos delincuenciales y que también pudieran, en el mejor de los casos, impedir el trabajo de los estudiosos de fronteras, y en el peor de los casos intimidarlos, confiscar sus equipos, y hasta someterlos al ejercicio de violencias verbales o físicas. Para quienes queremos que los estudiantes salgan al campo, es importante considerar todos estos riesgos adicionales.

\section{La fronterología y el activismo}

Sin duda, la relación entre el mundo académico y el activismo siempre ha sido complicada. Hay quienes consideran el mundo académico una torre de marfil, remota e inaccesible al mundo cotidiano. Es difícil olvidar por ejemplo que el escolasticismo medieval se hizo acreedor a la pregunta: ¿cuántos ángeles caben en la 
punta de un alfiler? Pero la realidad es que la relación entre la academia y el mundo que la rodea es mucho más compleja (Deshman y Hannah-Moffat, 2015) y ciertamente cada vez más estrecha. Los académicos, o por lo menos la mayoría, consideran sumamente importante aportar a partir de sus investigaciones científicas insumos para cambiar el entorno-ya sea a través de una interacción directa con los decisores en política pública, mediante la difusión directa del conocimiento generado hacia el público en general, o articulando cuestionamientos y tributando datos para los activistas de la sociedad civil (Staudt y Coronado, 2002; Coronado, 2019). En varias reuniones académicas hemos escuchado a colegas describir su trabajo de investigación que a veces les piden varias diferentes entidades del gobierno. Por ejemplo, en una conferencia en la sede de El Colegio de la Frontera Norte (COLEF) en Tijuana, se explicó en una mesa de trabajo que investigaciones que llevaban a cabo en ese centro de investigación, fueron promovidas e impulsadas por la Secretaria de Salud a nivel federal para poder documentar el beneficio de que madres que acaban de dar a luz se quedaran internadas 24 horas después del parto. La investigación indicó que las madres que salían antes de las 24 horas regresaban ellas y sus bebés con problemas más serios de salud. Las recomendaciones de una nueva política pública para que las madres se quedaran en el hospital las 24 horas dieron buenos resultados.

Una importante cantidad de académicos se convierten en funcionarios, comentaristas mediáticos, consultores, y hasta activistas. Los fronterólogos no son la excepción e incluso, entre quienes estudian las fronteras, se percibe más profundamente la necesidad de resistir los efectos más perversos de los procesos de fronterización-aunque hay que reconocer que hay quienes se conforman con ir al campo, investigar un tema, escribir sobre el mismo, y extraerse sin mucho más que un artículo publicado en alguna revista indexada. Para la mayor parte de los fronterólogos, sin embargo, esto no es el caso.

Muchos fronterólogos se convierten en observadores críticos de las fronteras y cuestionan de manera constante el aparato de seguridad, el trato inhumano y falto de ética de los migrantes, la ausencia de esquemas de cooperación para la gobernanza de las 
zonas fronterizas, la explotación capitalista de los trabajadores, los efectos del creciente comercio internacional en la habitabilidad de las fronteras, etc. Los autores de este artículo hemos visto de primera mano un acerbo emergente de obras que zigzaguean entre lo académico y el activismo. Esto evidentemente no es único de una frontera. Esta necesidad de justicia, ante las revelaciones de injusticia mediante estudios de campo, ocurre en muchas fronteras. Así como se dan en la frontera México-Estados Unidos, también ocurren en la frontera entre Israel y Palestina o con los flujos entre África e Italia o el Medio Oriente y Turquía y Grecia.

Ahora bien, las fronteras son, sin duda, espacios de enorme controversia en el mundo moderno. En las franjas fronterizas convergen la explotación económica y laboral, el tráfico de personas, los flujos ilegales que dan razón a gran parte de la delincuencia organizada, anillos de pobreza, administración competitiva e ineficiente de recursos naturales, burocracias represivas, cancelación de derechos procesales y hasta humanos, etc. Quienes estudian la gobernanza y la habitabilidad de las zonas y los flujos transfronterizos rápidamente caen en la cuenta de que las fronteras son fácilmente espacios de explotación, represión, injusticia, exclusión, etc. Ante estos fenómenos es difícil no convertirse en un activista. Pero esto presenta dos retos importantes.

El primer reto tiene que ver con el proceso de enseñanza-aprendizaje. ¿Cómo conducir al estudiante de fronteras por el método científico, que implica una cierta distancia emocional, un vaciamiento de valores y prejuicios, una observación fría de los fenómenos a la mano, y una claridad y neutralidad efectivamente académica y al mismo tiempo inculcar un involucramiento humano con el sujeto y el espacio fronterizo y los esquemas de represión y exclusión que representan las fronteras? El docente-investigador debe necesariamente buscar el equilibrio entre estos dos mundos que si bien son compatibles pueden fácilmente convertirse en mutuamente disruptivos. El segundo reto consiste en mantener una relación relativamente afable con los actores que habitan los espacios fronterizos a fin de conservar el acceso a sus datos, su conocimiento, y sus perspectivas y al mismo tiempo ejercer una conciencia social capaz de exponer las agendas más obscuras, de descubrir 
los esquemas de opresión y exclusión, de exhibir las estructuras de poder, de cuestionar al mismo Estado-nación y sus burocracias. Los académicos fronterizos-de manera muy intensa, aunque no necesariamente única-se encuentran por fuerza entre quienes ostentan y ejercen el poder y entre quienes son objeto de la ostentación y ejercicio de ese poder. Mantener la neutralidad que exige la academia y mantener un acceso permanente a los espacios objeto de estudio es sin duda una tarea que requiere de una habilidad fina en extremo. Otro reto es el compromiso que uno incurre cuando hace su investigación con organizaciones no gubernamentales (ONGs) en sitios como La Casa del Migrante ${ }^{3}$ y la organización Deported Veterans Support House-Bunker Juárez (Casa de Apoyo a Veteranos Deportados, etc., en los cuales el investigador siente, por ética y empatía, la necesidad de un quid pro quo. Uno de los autores de este ensayo, por ejemplo, al hacer trabajo de investigación con migrantes, por este tipo de situaciones, ha llevado donativos a La Casa del Migrante en Ciudad Juárez, y a los veteranos deportados les pidió que no dudaran en manifestar sus necesidades y que se iba a hacer todo lo posible por darle atención a su situación y apoyar para que el Congreso de EE. UU. aprobase alguna de las versiones de la iniciativa de ley titulada Return Our Veterans. Esto implica que, mientras que es importante formar estudiosos de las fronteras, es también difícil no inculcar

3 La misión de las Casas del Migrante Scalabrini es ofrecer respuestas y servicios de forma inmediata, gratuita y desinteresada promoviendo a la persona migrante de una manera integral (https://www.migrantes. com.mx/casas-del-migrante-scalabrinianas). Varias ciudades fronterizas, como Tijuana, Ciudad Juárez, Nuevo Laredo cuentan con una Casa del Migrante que apoyan a migrantes, refugiados y a deportados de los Estados Unidos.

4 Estados Unidos permite a residentes legales permanentes ingresar en el ejército, aunque no sean ciudadanos americanos. Varios residentes permanentes legales que nacieron en México han servido en el ejército americano. Algunos miembros de las fuerzas armadas con residencia legal permanente, después de su servicio militar en Viet Nam, Iraq, Afganistán, han tenido problemas legales y han sido deportados a su país de origen en este caso a México. Estos veteranos deportados han establecido "bunkers" en ciudades fronterizas, Mexicali, Ciudad Juarez, Tijuana, para apoyarse mutuamente. Ver: https://www.facebook.com/ DVSHJuarez/. 
cierta empatía por diversos actores que lidian con las fronteras y sus efectos día a día. La formación ética es tan importante como la formación intelectual y académica.

Lo que sí es cierto es que raramente se puede ser académico sin traspasar la línea del activismo en pro de quienes son las víctimas de los esquemas que hoy y cada vez más gobiernan las franjas fronterizas como espacios de excepción. Una de las más elocuentes exposiciones de estos dilemas es el trabajo de Ochoa O'Leary, Deeds, y Whiteford: Uncharted Terrains: New Directions in Border Research, Methodology and Practice (2013). Los autores de este texto exploran los puntos más centrales de este tema: la investigación en contextos transnacionales, frecuentemente impredecibles y volátiles y la pregunta central sobre la capacidad del académico de trabajar con poblaciones vulnerables, tales como los migrantes, por ejemplo, y mantener al mismo tiempo todos los estándares éticos y metodológicos mientras se intenta proveer información a los decisores sin incriminar a migrantes o coyotes o traficantes, etc. Además, toda la información generada por los académicos se puede convertir sin duda en insumos para una política fronteriza mucho más punitiva-ya sea en migración, trata de personas, o trabajadores de maquiladoras, o cualquier otro tema. Y si el académico se convierte en un activista, las autoridades a veces, y cada vez más, lo ponen en la mira e incluso lo penalizan, como lo fue el caso de Scott Warren en Arizona, quien fue preso, convicto, y después liberado bajo un proceso de apelación, por haber dedicado parte de sus esfuerzos a establecer estaciones de agua potable para los migrantes en el desierto de Arizona.

\section{La ética en los estudios fronterizos}

Uno de los retos del proceso de enseñanza-aprendizaje que merece atención en el estudio de las fronteras-como en muchos otros campos, especialmente en aquellos como la medicina-es el tema de la interacción entre el sujeto de estudio y el estudioso. Esto es una cuestión que tiene que ver con la ética (Ojeda de López et al., 2007). Efectivamente, así como los estudiosos de las fronteras a veces se convierten en activistas por entrar en contacto con las estructuras de poder con todas sus externalidades negativas que se 
manifiestan en las franjas fronterizas, estos también a menudo interactúan con personas que utilizan la frontera para sus fines, legales o ilegales. Al hacerlo, los fronterólogos a menudo se encuentran con personajes tales como narcotraficantes, tratantes de personas, $\mathrm{y}$ otros individuos a lo mínimo desagradables y a lo máximo criminales que permiten acceso a sus actividades bajo la condición de que el académico no delate estas actividades. En cierto sentido esto compromete la ética del mismo investigador, quien puede encontrarse en una situación de peligro o en una situación en la cual otras personas están en peligro, pero no puede hacer nada o la condición es que no haga nada. En este mismo tenor, es difícil inducir a los estudiantes a realizar trabajo de campo sin tener que abordar estos difíciles momentos del investigador de campo. Sin embargo, es necesario discutir estos potenciales problemas con los estudiantes antes de que puedan ocurrir. Si no se establecen los límites éticos o morales de la investigación se arriesga a que el estudiante quede inhabilitado para reconocer ciertas situaciones con oportunidad y para que tome decisiones pertinentes y adecuadas para la protección de su persona y la integridad de su sujeto de estudio - sin llegar a ser un cómplice de facto de una acción ilegal o inmoral, pero sin privarse del acceso a la oportunidad de observar el fenómeno de interés.

En resumen, el académico busca investigar el objeto de su interés desde un punto de vista científico, pero ¿cuál debe ser su relación humana con este objeto? Las preguntas son siempre las mismas: ¿Cómo entrar en un esquema de investigación y observar actividades cuestionables ya sea legal o moralmente y no intervenir? ¿Cómo proteger al sujeto de estudio - pregunta algo fácil de responder cuando se trata de una víctima, pero nada fácil de responder cuando se trata de un victimario? La respuesta reside, quizás, en un equilibrio satisfactorio a la conciencia del investigador, quien debe saber en qué momento y cómo balancear su interés científico con su obligación moral como ser humano y como persona.

\section{Los costos de estudiar la frontera}

Estudiar las fronteras tiene otro reto importante: La ubiquidad de las fronteras y los elevados costos que esto significa para llevar a cabo estudios comparados de fronteras. El objeto de es- 
tudio de los fronterólogos está en todos lados: zonas limítrofes entre países y territorios; puertos marítimos y aeropuertos; zonas oceánicas remotas; y cada vez más espacios fronterizados en el interior de regiones y países. Algunas fronteras son de remoto acceso o envueltas en conflictos armados o flujos transfronterizos a los cuales hay poco acceso. En este sentido, hacer trabajo de investigación es sumamente costoso. Y no hacer trabajo de campo es restarle credibilidad al trabajo del investigador- quien se vería confinado al trabajo de escritorio, el cual pierde los matices que sólo se pueden accesar estando en el terreno de investigación. Así pues, investigar las fronteras, por lo menos de manera comparada o masiva, requiere de una serie de recursos raramente disponibles en las ciencias sociales y ciertamente en la fronterología.

Ahora bien, los fronterólogos se encuentran en todo el mundo y, como en casi cualquier disciplina, interactuar con otros fronterólogos requiere de trabajo en conjunto tales como seminarios, conferencias, talleres, etc., los cuales se pueden tornar extremadamente caros rápidamente. Como muestra se encuentra el trabajo de los fronterólogos que se reúnen en el contexto de la Asociación de Estudios Fronterizos (ABS, por sus siglas en inglés) y de la red "Regiones Fronterizas en Transición" (BRIT, por sus siglas en inglés). La Asociación de Estudios Fronterizos se reúne una vez al año en alguna ciudad de los Estados Unidos. Cualquier participante debe estar preparado para pagar transporte aéreo, alojamiento, y otros viáticos, además de las cuotas de la asociación y de la conferencia. La presencia de la Covid-19 en el año 2020, sin embargo, ha abierto la posibilidad de llevar a cabo conferencias virtuales, que pudieran no sólo ser menos costosas sino más incluyentes. La oportunidad de asistir a interactuar con la comunidad epistémica de estudios fronterizos, y escuchar el trabajo de otros participantes y presentar el trabajo propio, puede costar hasta miles de dólares. Pero mucho de los gastos los pagan las universidades. Para los europeos esto pudiera ser un tanto asequible, pero para personas que tiene que viajar de Latinoamérica, África, y algunos países de Asia el costo es a menudo prohibitivo. En la experiencia de los autores de este ensayo, por ejemplo, llevar a los estudiantes de postgrado de las universidades mexicanas es difícil, no sólo por el costo sino 
porque también a veces hay dificultades para obtener visas, ya que muchas conferencias y talleres se llevan a cabo en países que requieren de tal documento para el ingreso. Es sorprendente que a pesar de todo los obstáculos muchos académicos de países donde las universidades tienen menores recursos participen en los congresos de la ABS. Lo mismo ocurre con las reuniones bienales de la red Regiones Fronterizas en Transición. Precisamente porque estas conferencias se llevan a cabo en una frontera del mundotales como Israel-Palestina, Corea-Japón, Nigeria-Benín, Alemania-Dinamarca, India-Bangladesh, etc.-las cantidades monetarias necesarias para desplazarse no sólo excluyen a los estudiantes de postgrado sino también a muchos académicos ya avanzados en sus carreras. De igual manera, existen una serie de centros de investigación fronteriza importantes, tales como el "Centro de Estudios Fronterizos" de la Universidad de Durham, en Inglaterra, el cual se enfoca en la resolución de disputas territoriales con un énfasis especial en la resolución de disputas marítimas. Los seminarios son altamente deseables, pero se llevan a cabo en Inglaterra, haciendo muy inaccesible sus conocimientos a personas de muchos países que pudieran interesarse en el tema ${ }^{5}$.

Vale la pena también hablar del tema del idioma. La lingua franca de los estudios fronterizos es el inglés. Pero muchas personas que estudiantes fronteras no hablan este idioma o no lo pueden leer, impidiéndose así el acceso a la bibliografía en ese idioma. Este problema sucede también en dirección opuesta. Muchos académicos de Estados Unidos no hablan español, portugués, francés, italiano o chino-idiomas con un creciente acervo de estudios de fronteras, pero que siguen inaccesibles a quienes no los leen. Entrenar a los estudiantes en otros idiomas es también difícil y caro. Por tanto, el desarrollo y los intercambios en la meta-disciplina quedan limitados por el problema de los idiomas, aunque esto es algo en común con otras disciplinas universales. En los estudios fronterizos, sin embargo, esto es un probado obstáculo para el avance de la meta-disciplina.

5 El IBRU: Centro para Investigaciones Fronterizas es parte del Departamento de Geografía de la Universidad de Durham, en Inglaterra. Más informaciones están disponibles en: https://www.dur.ac.uk/ibru. 
En general, para los fronterólogos, los costos involucran salarios tanto del investigador como del resto del equipo; materiales y equipo de investigación tales como las computadoras, las cámaras, las bases de datos, los instrumentos de medición, etc.; el tiempo dedicado al traslado, las reuniones, las conferencias, etc.; y otros costos del trabajo de campo, incluyendo la negociación del acceso a los datos, la construcción de redes, y los procesos de publicación. Hay otros costos no monetizados, por supuesto, tales como el tiempo lejos de casa o familia. Cualquier docente-investigador dedicado a la academia en la meta-disciplina de los estudios fronterizos debe considerar estos elementos al diseñar no sólo sus propias investigaciones sino también las investigaciones emprendidas por sus estudiantes.

\section{El reto de enseñar $\mathbf{y}$ aprender las fronteras en el siglo XXI}

Los estudios fronterizos son una meta-disciplina emergente que ha cobrado enorme importancia en años recientes. En este sentido, los estudios fronterizos no son diferentes de otras disciplinas; sus fortunas suben y bajan con los eventos contemporáneos coyunturales y de mediano y largo alcance. En los 1990s, la meta-disciplina pasó por un período de harto optimismo sobre los procesos de globalización y, sobre todo, de lo que parecía ser una oleada de procesos desfronterizantes - léase apertura de fronteras en todo el mundo, liderados en parte por los avances de la Unión Europea y acuerdos regionalizantes como el Tratado de Libre Comercio de Norteamérica (hoy Tratado México-Estados Unidos-Canadá, T-MEC). Después de los ataques terroristas del 11 de septiembre del 2001 en los Estados Unidos, la disciplina viró hacia el estudio de la complejidad de las fronteras a partir de los nuevos procesos de fronterización. $Y$ hoy, la meta-disciplina presta atención a los flujos transfronterizos irregulares, tales como la migración, y el uso de las tecnologías para vigilar y controlar las fronteras. Es decir, la fronterología es también parte de su contexto y su curso obedece, como muchas otras disciplinas, a las preocupaciones presentes.

Asimismo, el interés en las fronteras del mundo ha atraído a muchos académicos nuevos y fortalecido las organizaciones académicas y las redes epistémicas dedicadas al estudio de las fronteras. Pero con la extensión y la "normalización" de la meta-disciplina y su importancia han crecido también los costos de ejercerla 
en el mundo. Además de los retos internos a la meta-disciplina y todavía no resueltos como son los grandes problemas de conceptualización, teorización, y metodología, los accesos al objeto de estudio se han reducido, los riegos han aumentado, los costos han subido, y los esfuerzos requeridos de los docentes-investigadores para consolidar la meta-disciplina se han elevado. Formar nuevos fronterólogos, tanto a nivel pregrado como a nivel postgrado, exige hoy en día considerar todos los costos que hoy se han normalizado en la disciplina, y obliga a mirar a otras disciplinas y meta-disciplinas con la finalidad de entender cómo se han resuelto muchos de estos retos y problemas.

\section{Referencias}

AMBROSINI, Maurizio; CINALLI, Manlio; JACOBSON, David. (Eds). Migration, Borders and Citizenship: Between Policy and Public Spheres. New York, NY: Palgrave Macmillan, 2020.

AMELINA, Anna; NEGRITZ, Devrimsel D; FAIST, Thomas; SCHILLER, Nina Glick. Beyond Methodological Nationalism: Research Methodologies for Cross-Border Studies. New York, NY: Routledge, 2012.

BHABHA, Homi K. Nation and Narration. New York, NY: Routledge, 2013.

BOEHMER, Charles R.; MEDINA, Sergio Peña. The Determinants of Open and Closed Borders. Journal of Borderlands Studies, v. 27, n.3, p. 273-285, 2012

CORONADO, Irasema. Resilient Families amidst adversity in Colonias. , Universidad Nacional Autónoma de Mexico: Voices of Mexico, n. 108, 2019

CORREA-CABRERA, Guadalupe; KONRAD, Víctor. North American Borders in Comparative Perspective. Tucson, AZ: The University of Arizona Press, 2020.

DAVITTI, Daria. Biopolitical Borders and the State of Exception in the European Migration 'Crisis'. European Journal of International Law 29, n. 4 (November), p. 1173-1196, 2019.

DEL BOSQUE, Melissa. Border Agents Can Now Get Classified Intelligence Information. Experts Call That Dangerous. ProPublica (Noviembre 29), 2019. Ver: https://www.propublica.org/article/ border-agents-can-now-get-classified-intelligence-information-experts-call-that-dangerous. 
DESHMAN, Abby; HANNAH-MOFFAT, Kelly. Advocacy and Academia: Considering Strategies of Cooperative Engagement." Social Justice v. 42, n.2, p. 91-112, 2015.

DÍAZ, George T. Border Contraband: A History of Smuggling across the Rio Grande. Austin, TX: University of Texas Press, 2015.

DIENER, Alexander; HAGEN, Joshua. Borderlines and Borderlands: Political Oddities at the Edge of the Nation-State. New York, NY: Rowman \& Littlefield Publishers, 2010.

GERBER, James; MENDOZA COTA, Eduardo. Data for Mexico-U.S. Border Studies: A Comparison of Mexican and U.S. Data Collection and Distribution. In: PAYAN, Tony; CRUZ, Pamela L. (Eds.). Binational Commons: Institutional Development and Governance on the U.S.-Mexico Border. Tucson, AZ: University of Arizona Press, 2020.

HAN, Sallie; ANTROSIO, Jason. Walls, Fences and Barriers: Anthropology on the Border. Open Anthropology v.7, n.1 (Marzo), 2019. Ver: https://www.americananthro.org/StayInformed/OAArticleDetail.aspx?ItemNumber=24623.

HERBST, Jeffrey. The Creation and Maintenance of National Boundaries in Africa. International Organization, v. 43, n. 4 (Autumn), p. 673692, 1989.

HOLMES, Oliver; HOLDER, Josh. Gaza Border Protests: 190 killed and 28,000 injured in a year of bloodshed. The Guardian (Marzo 29), 2019. Ver https://www.theguardian.com/world/ng-interactive/2019/mar/29/a-year-of-bloodshed-at-gaza-border-protests.

JORDAN, Miriam. An Arizona Teacher Helped Migrants. Jurors Couldn't Decide If It Was a Crime. The New York Times (June 11), 2019. Ver https://www.nytimes.com/2019/06/11/us/scott-warren-arizona-deaths.html.

LAMONT, Michèle ; MOLNAR, Virág. The Study of Boundaries in the Social Sciences. Annual Review of Sociology, v. 28; 167-195, 2002.

MAGYAR-HAAS, Veronika. Ambivalent Concepts of the Border: Political Borders - Bodily Boundaries. Social Work and Society, v. 10, n. 2, p.1-13, 2012.

MANYIKA, James et al. Global Flows in a Digital Age: How Trade, Finance, People, and Data Connect the World Economy. United States: McKensey global Institute, 2014. Ver: https://www.mckinsey.com/ / 
media/McKinsey/Featured\%20Insights/Globalization/Global\%20 flows\%20in\%20a\%20digital\%20age/Global_flows_in_a_digital_age_ Full_report\%20March_2015.ashx.

MARTÍNEZ, Glen A. Perceptions of Dialect in a Changing Society: Folk Linguistics along the Texas-Mexico Border. Journal of Sociolinguistics, v. 7, n. 1, p. 38-49, 2003.

MAVROUDI, Elizabeth; NAGEL, Caroline. Global Migration: Patterns, Processes and Politics. New York, NY: Routledge, 2017.

MELO RIBEIRO, Daniel. Art and Cartography as a Critique of Borders. International Cartographic Association, 2017. Ver https://www. researchgate.net/publication/325186558_Art_and_cartography_as_a_ critique_of_borders.

NEWMAN, David. On Borders and Power: A Theoretical Framework. Journal of Borderlands Studies, v.18, n. 1, p. 13-25, 2003.

NIKOLIC, Zoran. Atlas of Unusual Borders: Discover Intriguing Boundaries, Territories and Geographical Curiosities. London, UK: Collins, 2019.

NWEIHED, Kalone G. Frontera y límite en su marco mundial. Una aproximación a la 'fronterología'. Caracas, Venezuela: Equinoccio: Ediciones de la Universidad Simón Bolívar, 1990.

OCHOA O'LEARY, Anna; DEEDS, Colin M; WHITEFORD, Scott. Uncharted Terrains: New Directions in Border Research, Methodology and Practice. Tucson, Arizona: University of Arizona Press, 2013.

OJEDA DE LÓPEZ, Juana; QUINTERO, Johana; MACHADO, Ineida. La ética en la investigación. Telos v. 9, n. 2 (Mayo-Agosto), p. 345-357, 2007.

PAASI, Anssi. Generations and the 'Development' of Border Studies. Geopolitics, v. 10, n. 4, p. 663-671, 2012.

PARK, Lisa Sun-Hee; PELLOW, David Naguib. Forum 4: The Environmental Privilege of Borders in the Anthropocene. Mobilities v. 14, n. 3, p. 395-400, 2019.

PARKER, Noel; VAUGHAN-WILLIAMS, Nick. Lines in the Sand? Toward an Agenda for Critical Border Studies. Geopolitics 14; 582-587, 2009.

PARKER, Noel; VAUGHAN-WILLIAMS, Nick. Critical Border Studies: Deepening the Lines in the Sand. Geopolitics, v.17, p. 727-733, 2012. 
PAYAN, Tony. Theory-Building in Border Studies: The View from North America. Eurasia Border Review, v. 5, n. 1. Hokkaido, Japón: Universidad de Hokkaido, p. 1-18, 2014.

POPESCU, Gabriel. Bordering and Ordering in the $21^{\text {st }}$ Century: Understanding Borders. New York, NY: Rowman and Littlefield Publishers, 2011.

ROTH, Michael. Global Organized Crime: A $21^{\text {st }}$ Century Approach. New York, NY: Routledge, 2017.

RUMFORD, Chris. Toward a Multiperspectival Study of Borders. Geopolitics, v. 17, p. 887-902, 2012.

SALTER, Mark B. When the Exception Becomes the Rule: Borders, Sovereignty and Citizenship. Citizenship Studies, v. 12, n. 4, (January), p. 365-380, 2008.

SCHNEIDER, Jacqueline. Sold into Extinction: The Global Trade in Endangered Species. Santa Barbara, CA: ABC-CLIO, 2012.

SEVASTIANOV, Sergei V.; LAINE, Jussi P.; KIREEV, Anton A. Introduction to Border Studies. Vladivostok, Rusia: Dalnauka, 2015.

STAUDT, Kathleen; CORONADO, Irasema. Fronteras No Mas: Toward Social Justice at the U.S.-Mexico Border New York: Palgrave Macmillan, 2002.

TORRES CARRAL, Guillermo. La meta-disciplina en la educación ambiental. Ra Ximhai: Revista de Sociedad, Cultural y Desarrollo Sustentable v. 2, n. 1 (enero-abril), p. 209-229, 2006.

VALLET, Elisabeth. Borders, Fences, and Walls: State of Insecurity? New York, NY: Routledge, 2014.

VAN HOUTUM, Henk. The Geopolitics of Borders and Boundaries. Geopolitics v. 10, p. 672-679, 2005.

VAN SCHENDEL, Willem. How Borderlands, Illicit Flows, and Territorial States Interlock. In: VAN SCHENDEL, Willem; ABRAHAM, Itty. (Eds.). Illicit Flows and Criminal Things: States, Borders, and the Other Side of Globalization. Bloomington: Indiana University Press, 2005 WILSON, Thomas M. DONNAN, Hastings. Borders and Border Studies. In: WILSON, Thomas M. DONNAN, Hastings. (Eds.). A Companion to Border Studies. Hoboken, NJ: Blackwell Publishing LTD, p. 1-25, 2012. ZUREIK, Elia; SALTER, Mark B. Global Surveillance and Policing: Borders, Security, and Identity. London, UK and New York, NY: Willan Publishing, 2013. 